\title{
«Ein Nachschlagewerk für alle»
}

Interview: Bruno Kesseli

Dr. med. et lic. phil., Chefredaktor

Mit dem compendium COMPACT ist seit kurzem wieder ein Arzneimittel-Nachschlagewerk in Buchform auf dem Markt, das sämtliche in der Schweiz zugelassenen Medikamente enthält. Ulrich Schaefer, Geschäftsführer der herausgebenden Firma HCI Solutions, und Gert Printzen, Mitglied des Zentralvorstandes der FMH, erläutern die Gründe für die Neulancierung.

Das "Arzneimittelkompendium» wurde 2013 in der gedruckten Form eingestellt. Drei Jahre später wird nun mit dem compendium COMPACT erneut ein Nachschlagewerk zu Medikamenten in Buchform auf den Markt gebracht. Weshalb dieser Sinneswandel?

Ulrich Schaefer: Nach der Einstellung der obligatorischen Print-Version konnte man davon ausgehen, dass der Wechsel von Print zu Online sehr schnell und prak-

«... war deshalb für uns die Sache klar:

Wir machen es - und zwar kompakt!»

tisch vollständig erfolgt. In der Realität sind heute aber an vielen Orten in Praxis, Spital und Pflege noch veraltete gedruckte Ausgaben von Kompendium und Brevier im Einsatz. Umfragen zeigen zudem, dass fast die Hälfte der Praxis-Ärzte ein Druckwerk vermisst. Als die Firma Zentiva sich bereit erklärte, einen grossen
Teil der Auflage abzunehmen, war deshalb für uns die Sache klar: Wir machen es - und zwar kompakt! Gert Printzen: Interessanterweise zeigten Umfragen, die die FMH lancierte, dass nicht nur die etwas älteren Kolleginnen und Kollegen an der Print-Version interessiert waren, sondern diesbezüglich auch seitens der jüngeren Bedarf angemeldet wurde.

\section{Herr Printzen, welche Rolle spielte die FMH bei der Neulancierung?}

Im Vorfeld hat sich die FMH einerseits über das Editorial in der Schweizerischen Ärztezeitung [1] «öffentlich» $\mathrm{zu}$ dieser Thematik geäussert und auf diese Weise eine Vielzahl von Antworten niedergelassener Kollegen und Kolleginnen erhalten, die uns baten, auf jeden Fall für eine Weiterführung des Kompendiums in schriftlicher Form einzutreten. Andererseits haben wir dieses Thema in unserer FMH-Arbeitsgruppe

\section{Die Interviewpartner}

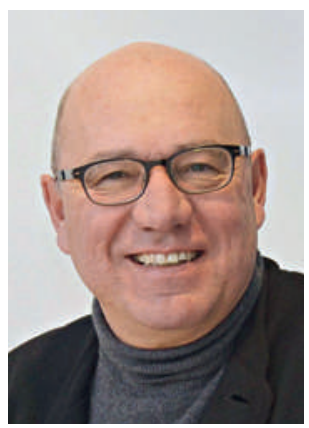

«Den schnellen Überblick garantieren»: Ulrich Schaefer über das compendium COMPACT.
Ulrich Schaefer ist Pharmazeut und dissertierte auf dem Gebiet der computerisierten Wirkstoff-Analyse.Seit 2011 ist er Geschäftsführer der Firma $\mathrm{HCl}$ Solutions AG, in welcher die Aktivitäten der bisherigen Firmen Documed, e-mediat und Triamun zusammengefasst sind. Arbeitsschwerpunkt ist die Medikationssicherheit in integrierten Systemen. Er ist Vorstandsmitglied der Interessengemeinschaften eHealth sowie eMediplan.

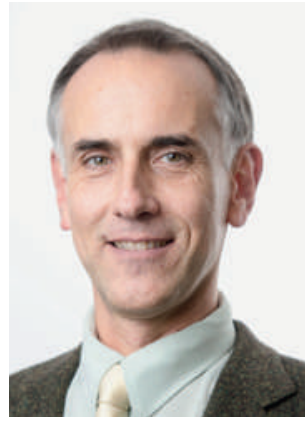

Ortet «Bedarf auch seitens der Jüngeren»: Gert Printzen.

Gert Printzen ist Facharzt für Labormedizin und verfügt auch über ein Universitätsdiplom in Biochemie. Seit 2010 ist er Mitglied des Zentralvorstandes der $\mathrm{FMH}$, in dem er die Departemente "eHealth - medizinische Informatik und Dokumentation», "Heilmittel» und "Paramedizinische Berufe» leitet. 
eHealth bearbeitet, die sich aus Vertretern der Basisorganisationen der Ärztekammer und somit schweizweit repräsentativ aus deren Interessenvertretern zusammensetzt. Und schliesslich haben wir auf politischer Ebene den Runden Tisch ins Leben gerufen und so alle Stakeholder zum Thema Arzneimittelkompendium und Patientensicherheit zusammengebracht und davon überzeugt, dass im Kompendium aktuelle vollständige Daten in strukturierter Form einzusetzen sind. Dies ist Basis für die elektronische Ausgabe, aber auch relevant für die Qualität der Schriftform.

Wie sieht das Konzept des compendium COMPACT aus und inwiefern unterscheidet es sich vom früheren Arzneimittelkompendium?

Schaefer: Die letzten Ausgaben des "alten" Kompendiums wurden aufgrund des vorgeschriebenen Textumfangs immer voluminöser - einzelne ArzneimittelMonographien umfassten mehrere engbeschriebene Seiten. Das neue, kompakte Kompendium enthält die wichtigsten Angaben pro Medikament in einer von unserer Fachredaktion gestrafften Form, um den schnellen Überblick zu garantieren.

«Wir sehen das neue Druckwerk als optimale Ergänzung zu den digitalen Plattformen - je nach Präferenz und Situation.»

Printzen: Es mag sein, dass das «extensive» vergangene Kompendium dem einen oder anderen fehlt, die neue Form liefert jedoch einen wichtigen ersten Überblick und wird beim Primärentscheid helfen.

Das Kompendium ist als compendium.ch im Internet zugänglich. Haben Sie Erkenntnisse darüber, wie und von wem es genutzt wird?

Schaefer: Praktisch alle Fachpersonen im Schweizer Gesundheitsmarkt nutzen die Online-Ausgabe und/ oder die mobilen Versionen. Aufgrund unserer regelmässigen Umfragen sind auch quantitative Aussagen möglich: So setzen zum Beispiel 80\% der Allgemeinmediziner das compendium.ch täglich ein.

Welche Zielgruppen sprechen Sie mit dem compendium COMPACT an? Sind es eher ältere Ärztinnen und Ärzte, die mit den modernen elektronischen Kommunikationsmitteln weniger vertraut sind?

Printzen: Wie eingangs erwähnt, hat das Editorial in der SÄZ viele direkte Reaktionen ausgelöst. Auch wenn das Gros der Bitten um Weiterführung des gedruckten

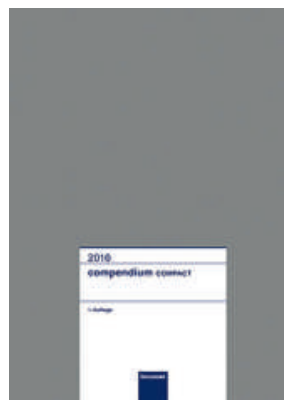

compendium COMPACT

Deutsche Ausgabe 2016. 1003 Seiten. Gebunden. sFr. 145.- / € (D) 145.ISBN 978-3-906819-01-3

ausging, besteht der Bedarf auch bei den Jungen. Eventuell erfolgt hierzu ja einmal eine Befragung. Schaefer: Ganz klar: Das compendium COMPACT ist ein Nachschlagewerk für alle. Es gibt nicht nur digital oder analog. Wir sehen das neue Druckwerk als optimale Ergänzung zu den digitalen Plattformen - je nach Präferenz und Situation.

In welchem Kontext sehen Sie die Nutzung des compendium COMPACT? Für die Kitteltasche ist es mit seinen 1000 Seiten kaum geeignet ...

Schaefer: Immer dann, wann es praktischer ist ... Printzen: Insbesondere bei «Downtime» oder Blockaden des Internets - oder sonstigen Übertragungsproblemen. Warten wir ab, welche Rückmeldungen wir bezüglich Dicke/Seitenzahl und Format erhalten.

\section{Ist es nicht absehbar, dass sich die elektronischen} Medien in Kürze auch bei der Ärzteschaft flächendeckend durchsetzen werden? Sehen Sie eine längerfristige Zukunft für die Printversion?

Printzen: Aufgrund der Rückmeldungen auf die FMHPublikationen sehe ich dieses Bedürfnis gleichwohl trotz Digital Natives.

Schaefer: Klar wird im Rahmen von eHealth die Nutzung elektronischer Medien auch in der Ärzteschaft zunehmen - aber wie gesagt: Die verschiedenen Formate ergänzen sich perfekt. Wichtiger als das Medium sind in jedem Fall die Zuverlässigkeit der Inhalte und deren anwenderfreundliche Präsentation.

Das Arzneimittelkompendium wurde einem Grossteil der Ärzteschaft direkt und gratis zugestellt. Wie gelangt das compendium COMPACT zu den Ärztinnen und Ärzten?

Schaefer: Im Gegensatz zu früher gibt es keinen Massenaussand an alle Praxen, Spitäler und Apotheken. Das compendium COMPACT kann beim Schweizerischen Ärzteverlag EMH oder bei der Zentiva AG bestellt werden. 